Check for updates

Cite this: RSC Adv., 2018, 8, 9031

\title{
Tunable graphene doping by modulating the nanopore geometry on a $\mathrm{SiO}_{2} / \mathrm{Si}$ substrate $\uparrow$
}

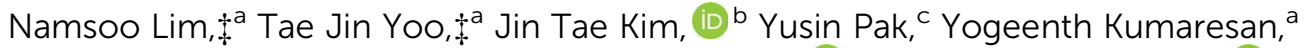 \\ Hyeonghun Kim, ${ }^{a}$ Woochul Kim, ${ }^{a}$ Byoung Hun Lee $\mathbb{D}^{\text {*a }}$ and Gun Young Jung (DD *a
}

A tunable graphene doping method utilizing a $\mathrm{SiO}_{2} / \mathrm{Si}$ substrate with nanopores (NP) was introduced. Laser interference lithography (LIL) using a He-Cd laser $(\lambda=325 \mathrm{~nm}$ ) was used to prepare pore size- and pitchcontrollable NP $\mathrm{SiO}_{2} / \mathrm{Si}$ substrates. Then, bottom-contact graphene field effect transistors (G-FETs) were fabricated on the NP $\mathrm{SiO}_{2} / \mathrm{Si}$ substrate to measure the transfer curves. The graphene transferred onto the $\mathrm{NP} \mathrm{SiO}_{2} / \mathrm{Si}$ substrate showed relatively $\mathrm{n}$-doped behavior compared to the graphene transferred onto a flat $\mathrm{SiO}_{2} / \mathrm{Si}$ substrate, as evidenced by the blue-shift of the $2 \mathrm{D}$ peak position $\left(2700 \mathrm{~cm}^{-1}\right)$ in the Raman spectra due to contact doping. As the porosity increased within the substrate, the Dirac voltage shifted to a more positive or negative value, depending on the initial doping type ( $p$ - or $n$-type, respectively) of the contact doping. The Dirac voltage shifts with porosity were ascribed mainly to the compensation for the reduced capacitance owing to the $\mathrm{SiO}_{2}$-air hetero-structured dielectric layer within the periodically aligned nanopores capped by the suspended graphene (electrostatic doping). The hysteresis (Dirac voltage difference during the forward and backward scans) was reduced when utilizing an $\mathrm{NP} \mathrm{SiO}_{2} / \mathrm{Si}$ substrate with smaller pores and/or a low porosity because fewer $\mathrm{H}_{2} \mathrm{O}$ or $\mathrm{O}_{2}$ molecules could be trapped inside the smaller pores.

Received 20th October 2017 Accepted 23rd February 2018

DOI: 10.1039/c7ra11601b

rsc.li/rsc-advances in a transparent electrode. ${ }^{13,14}$ The development of an effective doping technique for graphene is necessary to exploit these excellent properties of graphene in a wide range of (opto-)electronic devices. Graphene doping is crucial for realizing the $\mathrm{p} / \mathrm{n}$ junction, which is the basic unit for electronic circuits, ${ }^{15}$ and for the effective control of graphene's Fermi level for compatibility with other circuit elements. ${ }^{16}$

Graphene doping techniques have been explored with the following representative doping methods. ${ }^{17-19}$ The first type is electrostatic doping, which is the most common doping effect that occurs in the graphene field effect transistor (G-FET) structure. The main advantage of this approach is that the doping level is tunable by the gate potential without any implantation of additional dopants. The second type is contact doping. As graphene is a oneatom-thick two-dimensional $(2 \mathrm{D})$ material $(t \sim 0.34 \mathrm{~nm})$ that is very sensitive to its surroundings, it is easily doped by merely contacting a material; for example, graphene can be doped at $10^{11}$ charges per $\mathrm{cm}^{2}$ by contacting a $\mathrm{SiO}_{2}$ surface. ${ }^{20,21}$ The contact doping effect can be much enhanced in the case of a metal contact,

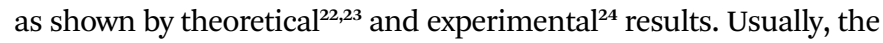
contact doping is an unavoidable phenomenon that is provided by the underlying substrate or during the electrode deposition. The third type is chemical doping. This effect is generated by the adsorbed molecules on the graphene surface in vapor or liquid states. Generally, compounds such as nitric acid, ${ }^{25}$ metal chlorides, organic molecules, ${ }^{26}$ and water ${ }^{1,27}$ are used as p-type dopants, and

compounds such as ammonia ${ }^{1,28}$ and $\mathrm{NO}_{2}$ (ref. 29-31) are used as

\footnotetext{
${ }^{a}$ School of Materials Science and Engineering, Gwangju Institute of Science and Technology (GIST), Gwangju, 500-712, Republic of Korea. E-mail: gyjung@gist.ac.kr; bhl@gist.ac.kr

${ }^{b}$ Creative Future Research Laboratory, Electronics and Telecommunications Research Institute, 218, Gajeong-ro Yuseong, Daejeon 305-700, Republic of Korea

${ }^{c}$ Physical Science and Engineering Division, King Abdullah University of Science and Technology (KAUST), Thuwal 23955-6900, Saudi Arabia

$\dagger$ Electronic supplementary information (ESI) available. See DOI: 10.1039/c7ra11601b

\$ The authors equally contributed to this work.
} 
n-type dopants. Finally, the fourth type is substitution doping. Here, some of the carbon atoms in the graphene lattice are replaced by other atoms (mainly boron (B) or nitrogen (N)), similar to the silicon-doping techniques. ${ }^{17,32,33}$ Using this technique, the bandgap of graphene can be opened; ${ }^{32}$ however, substitution doping leads to inevitable lattice breakages, resulting in lowquality graphene sheets. ${ }^{17}$

In this paper, we used $\mathrm{SiO}_{2} / \mathrm{Si}$ substrates having nanopores with different porosities and pitches ( $\mathrm{NP} \mathrm{SiO}_{2} / \mathrm{Si}$ substrate) to tune the doping state of the transferred graphene in contact with these substrates. Laser interference lithography (LIL) was used to prepare the pitch- and diameter-tunable nanopores. The doping state of the transferred graphene was characterized by Raman spectroscopy. Bottom-contact G-FETs were fabricated on the NP $\mathrm{SiO}_{2} / \mathrm{Si}$ substrates to observe the Dirac voltage shift due to the porosity-induced contact doping together with the $\mathrm{SiO}_{2}$-air heterostructured dielectric layer-induced electrostatic doping within the aligned nanopores. The effects of porosity and pitch on the Dirac voltage shift were also investigated. It was found that the direction of the Dirac voltage shift was determined by the initial doping polarity of graphene in contact with the underlying substrate.

\section{Experimental}

\section{Preparation of the $\mathrm{NP} \mathrm{SiO}_{2} / \mathrm{Si}$ substrates (Scheme 1)}

Laser interference lithography (LIL) was used to prepare the NP $\mathrm{SiO}_{2} / \mathrm{Si}$ substrates having different porosities at different pitches. A $300 \mathrm{~nm}$-thick $\mathrm{SiO}_{2} / \mathrm{Si}$ wafer (highly p-doped $\mathrm{Si}, \mathrm{QL}$ Electronics Co., China) was cleaned sequentially in acetone, isopropyl alcohol (IPA), and deionized water with sonication for
10 min. A $10 \mathrm{~nm}$-thick Cr layer was deposited on the substrate by an electron beam evaporator. Then, a bottom antireflection coating (BARC) layer (100 nm thick, AZ BARLi II 90, MicroChemicals, GmbH, Germany) and a negative photoresist (350 nm thick, AZ nLOF 2020, MicroChemicals GmbH, Germany) were spin-coated in sequence at $4000 \mathrm{rpm}$ for $40 \mathrm{~s}$ each. Each spin-coating step was followed by $1 \mathrm{~min}$ post-baking at $110^{\circ} \mathrm{C}$. The sample was double-exposed with a He-Cd laser $(\lambda=$ $325 \mathrm{~nm}$ ) and developed in a developing solution (AZ $300 \mathrm{MIF}$, MicroChemicals $\mathrm{GmbH}$, Germany) to produce an array of nanopores. The exposed BARC and $\mathrm{Cr}$ layers within the developed nanopores were selectively etched away by $\mathrm{O}_{2}$ plasma etching and with a Cr-wet etchant (CR-7, Cyantek corporation, USA) in sequence. Afterward, the photoresist and BARC layers were eliminated by immersing in a polymer remover (EKC 830, DuPont, USA), leaving the Cr nanopore array, which was used as a mask for etching of the underlying $\mathrm{SiO}_{2}$ with $\mathrm{CF}_{4}$ plasma. By eliminating the $\mathrm{Cr}$ mask with the $\mathrm{Cr}$ etchant, the $\mathrm{NP} \mathrm{SiO}_{2} / \mathrm{Si}$ substrate was finally produced.

\section{Fabrication of bottom-contact graphene field effect transistors (G-FETs)}

Bottom-contact graphene field effect transistors (G-FETs) were fabricated. The bottom-contact structure was suggested to minimize the damage to the transferred graphene. First, sourcedrain electrodes, Au (50 nm)/Ti $(10 \mathrm{~nm})$, were defined on the substrate by photolithography with a channel length of $10 \mu \mathrm{m}$ and a channel width of $50 \mu \mathrm{m}$. Afterward, single layer graphene (Graphene Square Inc., South Korea) was transferred onto the electrode-deposited substrate by the general wet-transfer

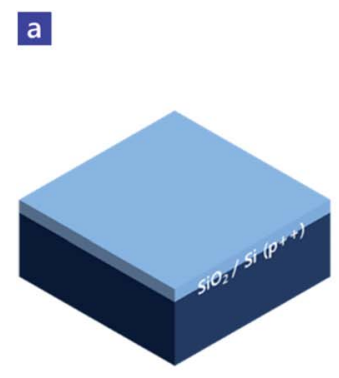

e

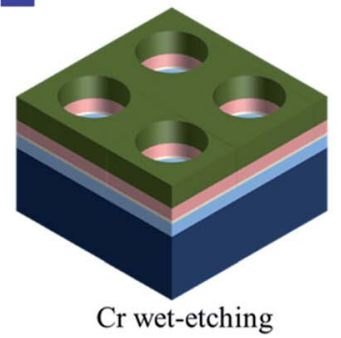

b

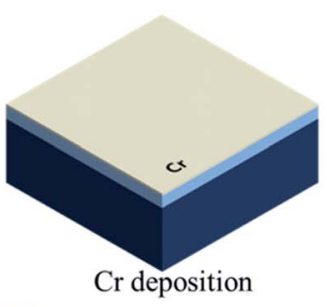

f

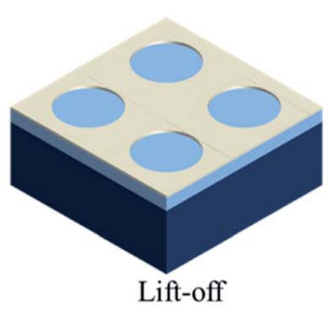

c

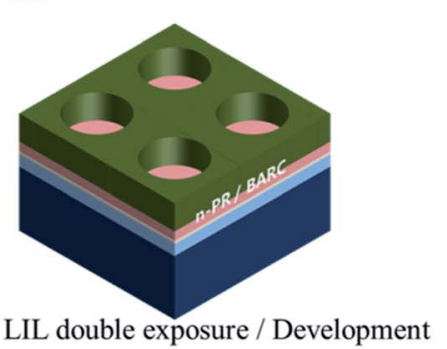

g

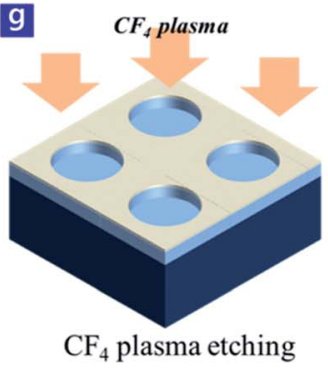

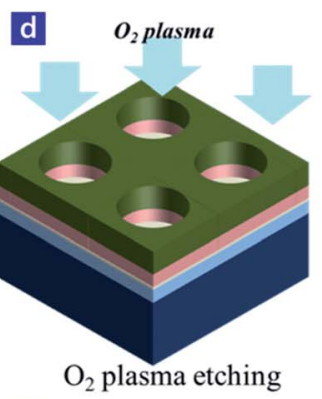

h

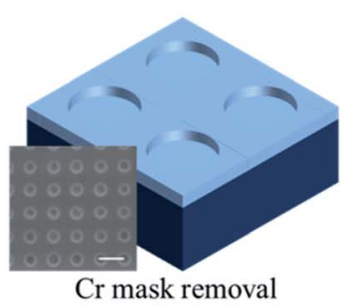

Scheme 1 Fabrication of the NP SiO $2 / S i$ substrate: (a) cleaning of the $\mathrm{SiO}_{2} / \mathrm{Si}$ substrate, (b) deposition of a $10 \mathrm{~nm}$ thick $\mathrm{Cr}$ layer, (c) spin-coating of BARC and n-PR layers for laser interference lithography, followed by double exposure with a He-Cd laser $(\lambda=325 \mathrm{~nm})$ and development process, resulting in the periodically aligned PR nanopores, (d) $\mathrm{O}_{2}$ plasma etching to eliminate the BARC and thus expose the underlying Cr layer

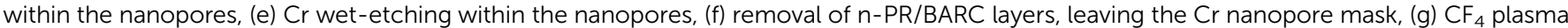
etching to transfer the $\mathrm{Cr}$ mask pattern to the underlying $\mathrm{SiO}_{2}$ layer, and (h) removal of the $\mathrm{Cr}$ mask to produce the final product of the $\mathrm{NP} \mathrm{SiO} / \mathrm{Si}$ substrate. The inset SEM image in (h) is the final $\mathrm{SiO}_{2} / \mathrm{Si}$ substrate having nanopores with a diameter of $442 \mathrm{~nm}$ at $1 \mu \mathrm{m}$ pitch (scale bar: $1 \mu \mathrm{m}$ ). 
technique. ${ }^{34}$ The transfer process is detailed in the ESI of S1. $\dagger$ The sample was then vacuum annealed at $300{ }^{\circ} \mathrm{C}$ for $1 \mathrm{~h}$. This annealing process for removing the transfer polymer or other impurities was essential to explore the substrate-induced graphene doping and strain effects. ${ }^{35}$ Finally, a $30 \mathrm{~nm} \mathrm{Al}_{2} \mathrm{O}_{3}$ layer was deposited on the whole sample as a passivation layer by atomic layer deposition (ALD) process.

\section{Measurement of the transfer characteristics $\left(I_{D}-V_{G}\right.$ curves)}

The $\mathrm{SiO}_{2}$ layer was partially scratched out with a diamond cutter, and the exposed highly p-doped silicon was used as the back-gate contact. A Keithley 4200 system together with a probestation (MST 5500B, MS TECH, South Korea) were utilized for measuring the transfer characteristics. The gate bias was introduced in the dual-sweep mode from $-50 \mathrm{~V}$ to $+50 \mathrm{~V}$. All measurements were performed inside a nitrogen-purged glove box.

\section{Results and discussion}

The schematic of the fabricated $\mathrm{NP} \mathrm{SiO}_{2} / \mathrm{Si}$ substrate is shown in Fig. 1a. The periodically aligned nanopores with a diameter $(d)$ at a pitch $(p)$ were produced by a laser interference lithography (LIL, $\lambda=325 \mathrm{~nm}$ ) process, which were controlled by several process parameters such as the Lloyd-mirror angle, laser exposure time and development time. The LIL system is further described in Fig. S2. $\dagger$ The pore height $(h, 150 \mathrm{~nm})$ was tuned by adjusting the plasma etching time with $\mathrm{CF}_{4}$ gas. Here, we fabricated $\mathrm{NP} \mathrm{SiO}_{2} / \mathrm{Si}$ substrates with three different pitches at $500 \mathrm{~nm}, 1000 \mathrm{~nm}$, and $1500 \mathrm{~nm}$ and three different pore diameters for each pitch. As the exposure time increased, the pore diameter decreased because a negative-tone photoresist (AZ nLOF 2020, MicroChemicals GmbH, Germany) was utilized, and thus, the porosity decreased.
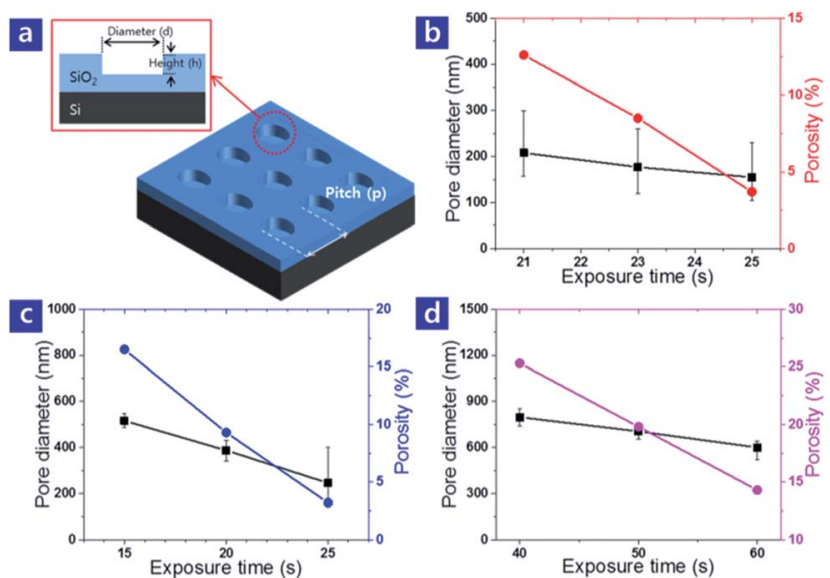

Fig. 1 (a) Schematic of the NP $\mathrm{SiO}_{2} / \mathrm{Si}$ substrate. Pore diameter and porosity vs. exposure time at different pitches of (b) $500 \mathrm{~nm}$, (c) $1000 \mathrm{~nm}$, and (d) $1500 \mathrm{~nm}$. The pitch was controlled by the interference angle between the two beams, and the pore diameter was determined by the exposure time and development time. All the samples were used as a gate dielectric layer for the following bottomcontact G-FETs.
Commercially available single layer graphene (Graphene Square Inc., South Korea) was transferred onto the produced various $\mathrm{NP} \mathrm{SiO}_{2} / \mathrm{Si}$ substrates by the wet-transfer technique. ${ }^{34}$ The obtained optical microscopic image shows that the transferred graphene was uniform and homogeneous without notable defects (Fig. 2a). Fig. 2b is a SEM image around the edge of the transferred graphene on the $\mathrm{NP} \mathrm{SiO}_{2} / \mathrm{Si}$ substrate with aligned nanopores at a $1000 \mathrm{~nm}$ pitch. The graphene was suspended on the nanopores (red-dotted circle), which were blurred due to the graphene coverage. The schematic image is the vertical view of a nanopore that suspends the transferred graphene. In contrast, the nanopores without graphene coverage showed a clear appearance.

Raman spectroscopy was utilized to see the effect of annealing, and the effect of the pores of the underlying substrate on the graphene doping. It was reported that during the annealing process, tensile strain of graphene layer was switched to compressive strain, which was indicated by the redshift of the Raman G- and 2D-peaks. ${ }^{36}$ Similar phenomena were occurred to our samples after heat-treatment even though the degree of red-shift was not large (see the ESI, S3†). Raman 2Dpeaks mapping of the graphene on the flat- and NP substrates at $1 \mu \mathrm{m}$ step in $10 \times 10 \mu \mathrm{m}^{2}$ area was also conducted before and after annealing process, indicating the less compressive strain of the suspended graphene on the nanopores (see the ESI, S4 $\dagger$ ). Fig. 2c compares the Raman spectra from the graphene transferred to flat and NP ( $d: 516 \mathrm{~nm}, p: 1000 \mathrm{~nm}) \mathrm{SiO}_{2} / \mathrm{Si}$ substrates. In the case of the graphene transferred on the $\mathrm{NP} \mathrm{SiO}_{2} / \mathrm{Si}$ substrate, the intensity of the D peak $\left(\sim 1350 \mathrm{~cm}^{-1}\right)$ increased compared to that for the flat $\mathrm{SiO}_{2} / \mathrm{Si}$ substrate. This could occur due to greater damage incurred during the graphene transfer onto the $\mathrm{NP} \mathrm{SiO}_{2} / \mathrm{Si}$ substrate. Another noticeable difference

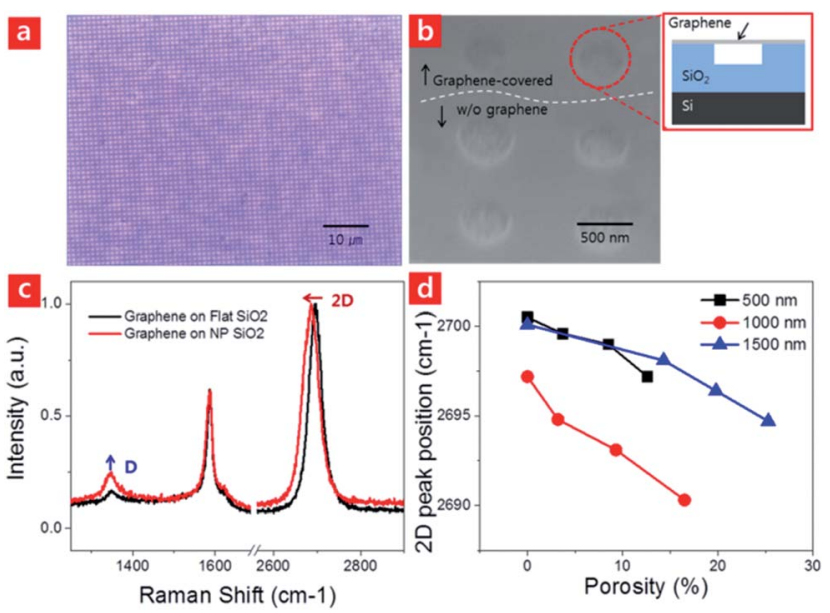

Fig. 2 (a) An optical microscope image of the transferred graphene. (b) A SEM image of the edge of transferred graphene on the NP $\mathrm{SiO}_{2} / \mathrm{Si}$ substrate. The nanopore (red circle) was blurred with the suspended graphene. Meanwhile, the nanopores outside the graphene coverage looked clear. The schematic image depicts the suspended graphene on a nanopore. (c) Comparison of the Raman spectra of the transferred graphene on the flat and $\mathrm{NP} \mathrm{SiO}_{2} / \mathrm{Si}$ substrates. (d) $2 \mathrm{D}$ peak shift in the Raman spectra of the transferred graphene vs. porosity of the $\mathrm{NP} \mathrm{SiO}_{2} /$ Si substrate at different pitches of $500 \mathrm{~nm}, 1000 \mathrm{~nm}$, and $1500 \mathrm{~nm}$. 
between the two Raman spectra is that the $2 \mathrm{D}$ peak position $\left(\sim 2700 \mathrm{~cm}^{-1}\right)$ of the graphene transferred on the $\mathrm{NP} \mathrm{SiO}_{2} / \mathrm{Si}$ substrate was relatively blue-shifted (from $2697 \mathrm{~cm}^{-1}$ to $2687 \mathrm{~cm}^{-1}$ ), signaling the relative n-doping phenomenon.

The graphene interfacing the oxygen (O)-terminated $\mathrm{SiO}_{2}$ substrate is known to be slightly p-doped by electron withdrawal from the graphene to the electronegative oxygen atom-

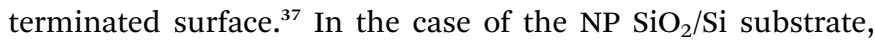
when graphene was transferred onto the substrate, the graphene was partially suspended at the pores, inducing less interfacial area between them. Therefore, relative n-doping of the overlaid graphene could occur, considering the reduced interfacial area with the $\mathrm{SiO}_{2}$ layer. The $2 \mathrm{D}$ peak positions of the Raman spectra were examined with the transferred graphene on $\mathrm{NP} \mathrm{SiO}_{2} / \mathrm{Si}$ substrates having three different porosities at a certain pitch (three different pitches in each pitch, total of 9 samples) to confirm the above hypothesis. Five points were measured in each sample. The Raman spectra from all the samples are shown in Fig. S5, $\dagger$ and the 2D peaks were collected to observe the doping tendency. At an identical pitch, as the porosity increased, the blue-shift degree of the $2 \mathrm{D}$ peak gradually increased, demonstrating the increment of the n-doping effect (Fig. 2d). Bottom-contact G-FETs were fabricated as shown in Fig. 3a to check the doping state of transferred graphene by measuring the Dirac voltage. Top-contact G-FETs were also prepared but, the underneath graphene was impaired during the source/drain electrode fabrication processes such as photolithography and the subsequent plasma etching. The topcontact G-FET made on the $\mathrm{NP}_{\mathrm{SiO}_{2}} / \mathrm{Si}$ substrates showed one order lower drain-current $\left(I_{\mathrm{D}}\right)$ level, compared to that from the flat $\mathrm{SiO}_{2} / \mathrm{Si}$ substrate (Fig. S6 $\dagger$ ); this was possibly due to more severe damages because the partially suspended graphene was not fully supported by the substrate. With this reason, bottomcontact G-FET structure was adopted to minimize the process steps above graphene. After graphene transfer, an $\mathrm{Al}_{2} \mathrm{O}_{3}$ passivation layer (30 $\mathrm{nm}$ thick) was deposited by atomic layer deposition (ALD) to passivate the graphene layer from the ambient atmosphere. Fig. $3 \mathrm{~b}$ shows a top-view SEM image of the
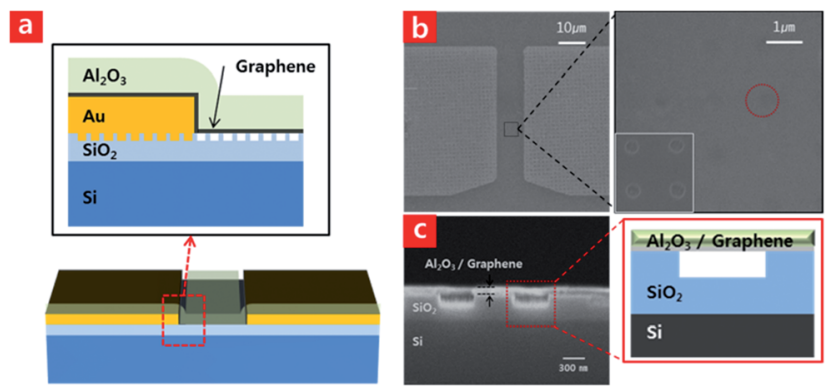

Fig. 3 (a) A schematic of the bottom-contact G-FET fabricated on the $\mathrm{NP} \mathrm{SiO}_{2} / \mathrm{Si}$ substrate. (b) A top-view SEM image of the G-FET on the NP $\mathrm{SiO}_{2} / \mathrm{Si}$ substrate. The channel region is magnified and compared with the inset image of the bare NP $\mathrm{SiO}_{2} / \mathrm{Si}$ substrate at the same magnification. The red circle indicates a vaguely seen nanopore on which the $\mathrm{Al}_{2} \mathrm{O}_{3} /$ graphene layers is suspended. (c) A cross-sectional SEM image together with an equivalent schematic in the red square area, revealing that the suspended $\mathrm{Al}_{2} \mathrm{O}_{3}$ /graphene layers were not sagging within the nanopore.
G-FET as well as a magnified image of $\mathrm{Al}_{2} \mathrm{O}_{3}$ /graphene on top of the NP substrate at the channel region. In addition, an image of the bare $\mathrm{NP} \mathrm{SiO}_{2} / \mathrm{Si}$ substrate with the same magnification is inset for comparison. The $\mathrm{Al}_{2} \mathrm{O}_{3}$ /graphene layer was suspended on the nanopores without noticeable breakages, and thus, the nanopores appeared to be blurred with the suspended layers. This was reconfirmed by the cross-sectional SEM image (Fig. 3c), indicating that the $\mathrm{Al}_{2} \mathrm{O}_{3}$ /graphene layer was well suspended without sagging within the nanopore.

Fig. 4 shows typical transfer curves of the G-FETs fabricated on the (a) flat and (b) $\mathrm{NP} \mathrm{SiO}_{2} / \mathrm{Si}$ substrate. A NP $\mathrm{SiO}_{2} / \mathrm{Si}$ substrate with nanopores at a $1000 \mathrm{~nm}$ pitch with $15 \%$ porosity was used. Hereafter, we define the Dirac voltage as the gate voltage at which the drain current is at the minimum during the gate-voltage sweep, and the hysteresis as the difference between the two Dirac voltages in forward and backward sweeps. In the case of flat $\mathrm{SiO}_{2} / \mathrm{Si}$ substrate, the Dirac voltages in forward and backward sweeps were nearly same independent of the drain voltages $\left(V_{\mathrm{D}}\right)$, and therefore, the hysteresis was minimal as

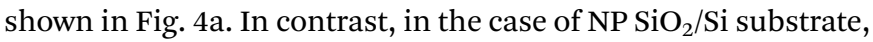
the Dirac voltages in both sweeps were much different, leading to enlarged hysteresis, as shown in Fig. $4 \mathrm{~b}$.

Table 1 summarizes the Dirac voltages and the hysteresises extracted from all transfer curves measured at different $V_{\mathrm{D}}$ of $0.1 \mathrm{~V}, 0.3 \mathrm{~V}$, and $0.5 \mathrm{~V}$. In the case of $\mathrm{NP} \mathrm{SiO}_{2} / \mathrm{Si}$ substrate, as the $V_{\mathrm{D}}$ increased, the Dirac voltage in backward sweep shifted from $-9 \mathrm{~V}$ to $-7 \mathrm{~V}$ along with the increased hysteresis from $8 \mathrm{~V}$ to $10 \mathrm{~V}$. It is reported that the hysteresis was mainly attributed to the $\mathrm{H}_{2} \mathrm{O}$ and $\mathrm{O}_{2}$ molecules trapped on graphene, generating $\mathrm{OH}^{-}$ions on the graphene (eqn (1)). ${ }^{38}$

$$
\mathrm{O}_{2}+2 \mathrm{H}_{2} \mathrm{O}+4 \mathrm{e}^{-} \text {(graphene) } \rightarrow 4 \mathrm{OH}^{-}
$$

In the forward sweep, when a gate voltage is positively biased, these $\mathrm{OH}^{-}$ions are accumulated at the interface between the $\mathrm{SiO}_{2}$ surface and the overlaid graphene owing to electrostatic effect, which partially screen the electric field between the gate electrode and the graphene in the following backward sweep. Thus, a higher gate voltage was required for the Fermi level to be aligned with the Dirac point, transiently shifting the Dirac voltage to the p-doping direction compared to that of forward sweep. ${ }^{38}$ In the case of the $\mathrm{NP} \mathrm{SiO}_{2} / \mathrm{Si}$ substrate, there are many nanopores that could trap more $\mathrm{H}_{2} \mathrm{O}$ and $\mathrm{O}_{2}$
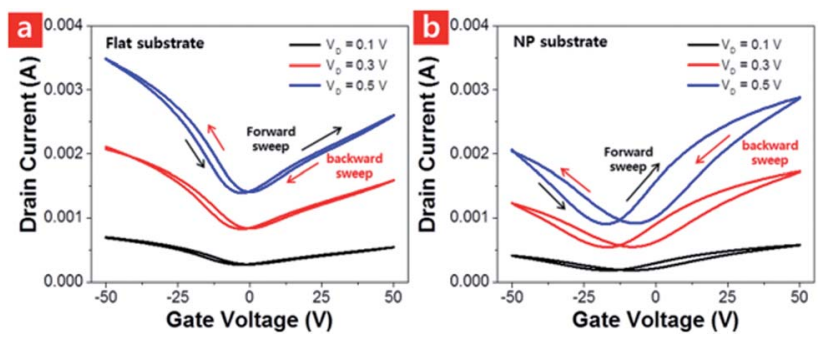

Fig. 4 Transfer characteristics ( $V_{G}-I_{D}$ curves) of the G-FETs produced on the (a) flat- and (b) NP $\mathrm{SiO}_{2} / \mathrm{Si}$ substrate. The gate voltage $\left(V_{\mathrm{G}}\right)$ was dual-swept from $-50 \mathrm{~V}$ to $+50 \mathrm{~V}$ at different drain voltages $(0.1 \mathrm{~V}$, $0.3 \mathrm{~V}$, and $0.5 \mathrm{~V}$ ). 
Table 1 Dirac voltages and hysteresises in the $V_{G}-I_{D}$ curves

\begin{tabular}{|c|c|c|c|c|c|c|}
\hline \multirow[b]{2}{*}{$V_{\mathrm{D}}[\mathrm{V}]$} & \multicolumn{3}{|c|}{ Flat $\mathrm{SiO}_{2} / \mathrm{Si}$ substrate } & \multicolumn{3}{|c|}{$\underline{\mathrm{NP} \mathrm{SiO}_{2} / \mathrm{Si} \text { substrate }}$} \\
\hline & FS & BS & Hysteresis & FS & BS & Hysteresis \\
\hline 0.1 & -3 & 0 & 3 & -17 & -9 & 8 \\
\hline 0.3 & -3 & 0 & 3 & -17 & -8 & 9 \\
\hline 0.5 & -3 & 0 & 3 & -17 & -7 & 10 \\
\hline
\end{tabular}

FS: forward sweep $(-50 \rightarrow+50 \mathrm{~V})$, BS: backward sweep $(+50 \rightarrow-50 \mathrm{~V})$.

molecules, inducing the larger hysteresis. In addition, the plasma-etching process used to make the nanopores generated a rougher surface that could supply more trap sites.

It is notable that the Dirac voltage of the $\mathrm{NP} \mathrm{SiO}_{2} / \mathrm{Si}$ substrate in the forward sweep shifted to a much more negative voltage $(-17 \mathrm{~V})$, compared to the $-3 \mathrm{~V}$ of the flat $\mathrm{SiO}_{2} / \mathrm{Si}$ substrate. This large relative $\mathrm{n}$-doping could not be explained by the contact doping effect only. Rather, this phenomenon can be explained by the electrostatic doping effect originated from the difference in the capacitance of dielectric layer. Fig. 5 shows the schematics of the dielectric layer of the two cases and their equivalent capacitance circuits for the (a) flat and (b) $\mathrm{NP} \mathrm{SiO}_{2} / \mathrm{Si}$ substrates. For the general metal-insulator-metal (MIM) capacitor structure, the capacitance $(C)$ is generally expressed by eqn (2);

$$
C=\varepsilon \frac{A}{d}
$$

Here, $\varepsilon, A$, and $d$ are the permittivity of the dielectric material, the overlapped area of the two plates, and the separation between the plates, respectively. For the flat $\mathrm{SiO}_{2} / \mathrm{Si}$ substrate, the capacitance is simply expressed as the overlapped equivalent capacitor circuit, as shown in Fig. 5a. For the $\mathrm{NP} \mathrm{SiO}_{2} / \mathrm{Si}$ substrate, it is complicated to calculate the capacitance because the total capacitance consists of two kinds of capacitances, as depicted in Fig. 5b. The first one is the 300 nm-thick $\mathrm{SiO}_{2}$ capacitance from the areas of supported graphene (red dotted rectangles), and the second is the capacitance from the areas of suspended graphene (blue dotted rectangles within the nanopores) in which the $150 \mathrm{~nm}$-thick $\mathrm{SiO}_{2}$ capacitance is serially connected to the $150 \mathrm{~nm}$-thick air capacitances $\left(\mathrm{SiO}_{2}\right.$-air heterostructured dielectric layer). Overall, these two capacitances are connected in parallel. The capacitance of the $300 \mathrm{~nm}$-thick $\mathrm{SiO}_{2}$ is known to be $11.6 \mathrm{nF} \mathrm{cm}^{-2} \cdot{ }^{39}$ From this value, the capacitances of the $150 \mathrm{~nm}$-thick $\mathrm{SiO}_{2}$ and the $150 \mathrm{~nm}$-thick air were
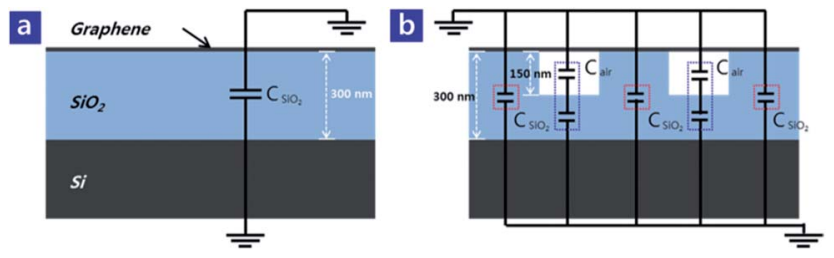

Fig. 5 Schematic of the cross-sectional dielectric layers along with their equivalent capacitor circuits for the (a) flat $\mathrm{SiO}_{2} / \mathrm{Si}$ substrate and (b) $\mathrm{NP} \mathrm{SiO}_{2} / \mathrm{Si}$ substrate. calculated as $23.2 \mathrm{nF} \mathrm{cm}^{-2}$, and $5.9 \mathrm{nF} \mathrm{cm}^{-2}$, respectively, by using $\varepsilon_{\text {air }}=1.0$ and $\varepsilon_{\mathrm{SiO}_{2}}=3.9$.

The capacitance calculation for the $\mathrm{NP} \mathrm{SiO}_{2} / \mathrm{Si}$ case is complicated and detailed in the ESI of S7. $\dagger$ Although it appears that many capacitances (all belonging to one of the two capacitance types) are connected in parallel to each other, the capacitance can be calculated simply by adding the two types of capacitances (calculated per unit area of $\mathrm{cm}^{2}$ ) multiplied by the respective porous (i.e., suspended graphene) and non-porous (i.e., supported graphene) area fractions; the calculated capacitances of the different samples having different pitch and porosity are listed in Table 2 . Compared to the capacitance of the flat $\mathrm{SiO}_{2} / \mathrm{Si}$ substrate $\left(11.6 \mathrm{nF} \mathrm{cm}{ }^{-2}\right)$, the calculated capac-

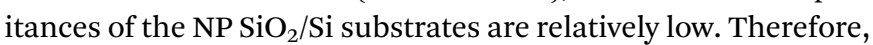
even though the same gate bias was introduced, the induced electric field to the graphene channel through the dielectric layer was lowered by the lower capacitance of the dielectric layer compared to that of the flat substrate. Consequently, in the case of NP G-FET, the Dirac voltage shifted to a more negative value than that of the flat G-FET to compensate for the reduced capacitance.

G-FETs were fabricated on the flat $\mathrm{SiO}_{2} / \mathrm{Si}$ substrate and on nine different $\mathrm{NP} \mathrm{SiO}_{2} / \mathrm{Si}$ substrates, as depicted in Fig. 1, to examine the effects of porosity and pitch on the Dirac voltage shift. Fig. 6a shows the representative transfer curves (zoomed in around the Dirac voltage) of the G-FETs fabricated on the 500 nm-pitch $\mathrm{NP} \mathrm{SiO}_{2} / \mathrm{Si}$ substrates with different porosities. The arrows indicate the Dirac voltages; black for the forward sweep and red for the backward sweep. Normally p-typed graphene became n-type when being contacted with the $500 \mathrm{~nm}$-pitch NP $\mathrm{SiO}_{2} / \mathrm{Si}$ substrate at $2 \%$ porosity. As the porosity increased, the Dirac voltage shifted to more negative value (Fig. 6b). The capacitance of the dielectric layer was reduced with increasing porosity (Table 2), leading to the Dirac voltage shift to more negative value to compensate for the reduced capacitance, as discussed in Fig. 5.

The transfer curves from the NP G-FETs with different porosities at $1000 \mathrm{~nm}$ - and $1500 \mathrm{~nm}$ pitches are shown in Fig. $\mathrm{S} 8, \dagger$ and the Dirac voltage shift with increasing porosity is plotted in Fig. $6 \mathrm{c}$ and d, respectively. Interestingly, for the case of the $1500 \mathrm{~nm}$ pitch, the Dirac voltage shifted to more positive value with increasing porosity (Fig. 6d), which is opposite to the case of the $500 \mathrm{~nm}$ pitch. We found that the direction of the Dirac voltage shift was mainly dependent on the initial doping polarity of the graphene (whether p-type or n-type) by the substrate-induced contact-doping. As discussed in the Raman

Table 2 Calculated capacitances of the various dielectric layers

\begin{tabular}{|c|c|c|c|c|c|c|}
\hline \multicolumn{3}{|c|}{ Flat } & \multicolumn{4}{|c|}{ Pitch: $1500 \mathrm{~nm}$} \\
\hline Porosity [\%] & 0 & 9.6 & & 15.0 & 21.7 & \\
\hline \multirow[t]{2}{*}{ Cap. $\left[\mathrm{nF} \mathrm{cm}^{-2}\right]$} & 11.6 & 10.94 & & 10.57 & 10.11 & \\
\hline & \multicolumn{2}{|c|}{ Pitch: $1000 \mathrm{~nm}$} & & \multicolumn{2}{|c|}{ Pitch: $500 \mathrm{~nm}$} & \\
\hline Porosity [\%] & 9.9 & 13.9 & 25.0 & 2.0 & 4.1 & 20.8 \\
\hline Cap. $\left[\mathrm{nF} \mathrm{cm}^{-2}\right]$ & 10.92 & 10.65 & 9.89 & 11.46 & 11.32 & 10.17 \\
\hline
\end{tabular}



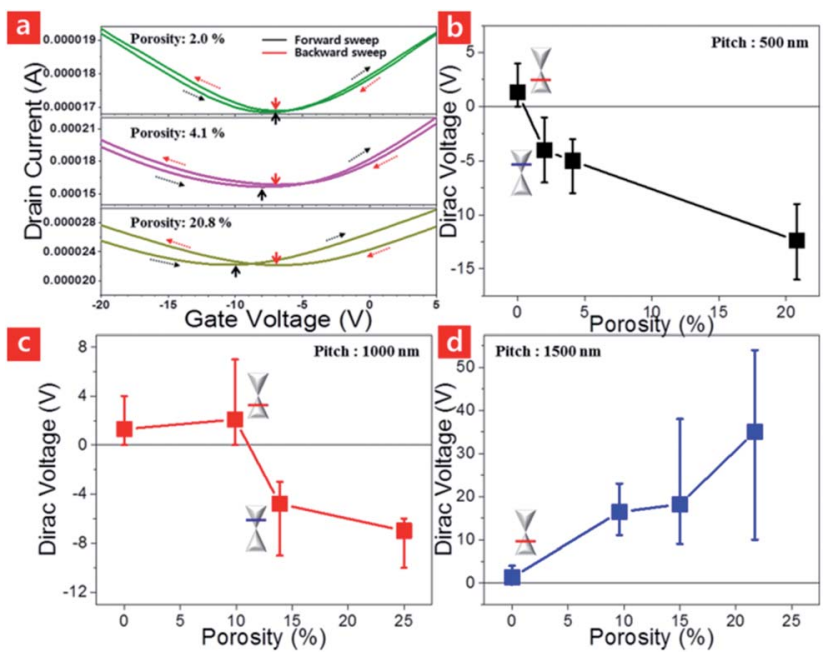

Fig. 6 (a) Comparison of the transfer curves (zoomed in around the Dirac voltage) of the G-FETs on the $\mathrm{NP} \mathrm{SiO}_{2} / \mathrm{Si}$ substrate at a pitch of $500 \mathrm{~nm}$; the arrows indicate the Dirac voltages during the forward(black) and backward sweep (red). The Dirac voltage shift vs. different porosities at a pitch of (b) $500 \mathrm{~nm}$, (c) $1000 \mathrm{~nm}$, and (d) $1500 \mathrm{~nm}$.

spectra results (Fig. 2c and d), the graphene on the $\mathrm{NP}^{\mathrm{SiO}_{2}} / \mathrm{Si}$ substrate demonstrated relative n-doping properties compared to the flat $\mathrm{SiO}_{2} / \mathrm{Si}$ substrate's case. If the graphene was initially n-type after contacting to the underneath substrate, the Dirac voltage shifted to a more negative value with increasing porosity, as in the case of $500 \mathrm{~nm}$ (Fig. 6b). If the graphene was initially p-type, on the other hand, the Dirac voltage shifted to a more positive value with increasing porosity, as in the case of $1500 \mathrm{~nm}$ (Fig. 6d).

In the case of $1000 \mathrm{~nm}$ pitch, the doping polarity switched at a certain porosity between $9.9 \%$ and $13.9 \%$, meaning that the transferred graphene was still p-type when it was contacted to the $\mathrm{SiO}_{2} / \mathrm{Si}$ substrate with the porosity below $9.9 \%$ and became n-type on top of the substrate having a porosity above $13.9 \%$ (Fig. 6c). Depending on the initial graphene doping polarity, different Dirac voltage shift was demonstrated with increasing the porosity; to a higher (positive) voltage up to $9.9 \%$ porosity with the initially p-type graphene or to a lower (negative) voltage above $13.9 \%$ porosity with the initially n-type graphene. This
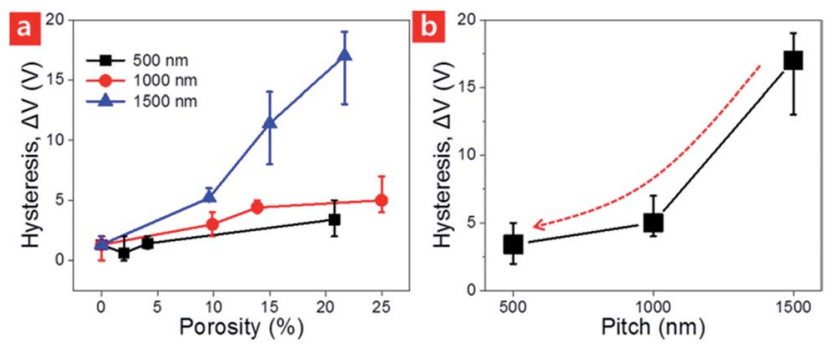

Fig. 7 (a) Hysteresis variation of the G-FETs on the $\mathrm{NP} \mathrm{SiO}_{2} / \mathrm{Si}$ substrates with different porosities at a fixed pitch. (b) Hysteresis variation of the G-FETs on the NP $\mathrm{SiO}_{2} / \mathrm{Si}$ substrates with different pitches, having similar porosities of $20.8 \%, 25 \%$, and $21.7 \%$ for the $500 \mathrm{~nm}, 1000 \mathrm{~nm}$, and $1500 \mathrm{~nm}$ pitch, respectively. result indicates that the initial graphene doping type can be modulated by the porosity of the underlying substrate.

Fig. 7a shows the effect of porosity on the hysteresis. At an identical pitch, an increase in porosity means that larger pores support the graphene layer, which could trap more $\mathrm{H}_{2} \mathrm{O}$ and $\mathrm{O}_{2}$ molecules, and thus generate more $\mathrm{OH}^{-}$ions, leading to a larger hysteresis, as shown in Fig. 7a. In addition, three samples with similar porosities were selected from each pitch to evaluate the pitch size effect on the hysteresis (Fig. 7b). In this case, a decrease in the pitch means that a graphene layer is suspended on highly dense nanopores with a smaller pore size. Therefore, a smaller amount of $\mathrm{H}_{2} \mathrm{O}$ or $\mathrm{O}_{2}$ molecules can be trapped inside the pores, inducing the reduced hysteresis with decreasing pitch size.

\section{Conclusions}

Substrate-induced doping effects were investigated with G-FETs fabricated on size- and pitch-tunable $\mathrm{NP} \mathrm{SiO}_{2} / \mathrm{Si}$ substrates. The graphene transferred onto the $\mathrm{NP} \mathrm{SiO}_{2} / \mathrm{Si}$ substrate was partially suspended on the nanopores and demonstrated relative ndoping properties compared to the transferred graphene on a flat $\mathrm{SiO}_{2} / \mathrm{Si}$ substrate. The dielectric layer composed of the periodically aligned nanopores had a relatively lower capacitance than that of the flat $\mathrm{SiO}_{2} / \mathrm{Si}$ substrate. The initial doping type of graphene was determined by the pore geometry on which the graphene was transferred. As the porosity increased, the Dirac voltage shifted to a higher positive or lower negative value, depending on the initial graphene doping polarity (p- or $\mathrm{n}$-type, respectively). The degree of graphene doping was affected by the combination of the contact and electrostatic effects. The $\mathrm{NP} \mathrm{SiO}_{2} / \mathrm{Si}$ substrate with smaller nanopores demonstrated a reduced hysteresis due to the smaller amount of $\mathrm{H}_{2} \mathrm{O}$ or $\mathrm{O}_{2}$ molecules trapped within the smaller pores.

This newly developed substrate-induced graphene doping technique has its worth in that graphene doping type can be effectively tuned by the nanopore geometry of the underlying substrate without additional dopants. This doping technique can also be applied to other two-dimensional (2D) materials, such as molybdenum disulfide $\left(\mathrm{MoS}_{2}\right)$, tungsten disulfide $\left(\mathrm{WS}_{2}\right)$, and tungsten diselenide (WSe ${ }_{2}$ ) for various applications.

\section{Conflicts of interest}

There are no conflicts to declare.

\section{Acknowledgements}

This work was supported by the Pioneer Research Center Program (NRF-2016M3C1A3908893) and by the Basic Science Research Program (NRF-2016R1A2B4006395) through the National Research Foundation of Korea (NRF) funded by the Ministry of Education. The research was partially supported by the GIST Research Institute (GRI) project through a grant provided by GIST in 2017. 


\section{Notes and references}

1 K. S. Novoselov, A. K. Geim, S. V. Morozov, D. Jiang, Y. Zhang, S. V. Dubonos, I. V. Grigorieva and A. A. Firsov, Science, 2004, 306, 666.

2 K. I Bolotin, K. J. Sikes, Z. Jiang, M. Klima, G. Fudenberg, J. Hone, P. Kim and H. L. Stormer, Solid State Commun., 2008, 146, 351.

3 L. A. Jauregui, Y. Yue, A. N. Sidorov, J. Hu, Q. Yu, G. Lopez, R. Jalilian, D. K. Benjamin, D. A. Delk, W. Wu, Z. Liu, X. Wang, Z. Jiang, X. Ruan, J. Bao, S. S. Pei and Y. P. Chen, ECS Trans., 2010, 28, 73.

4 C. Lee, X. Wei, J. W. Kysar and J. Hone, Science, 2008, 321, 385.

5 R. R. Nair, P. Blake, A. N. Grigorenko, K. S. Novoselov, T. J. Booth, T. Stauber, N. M. R. Peres and A. K. Geim, Science, 2008, 320, 1308.

6 K. F. Mak, L. Ju, F. Wang and T. F. Heinz, Solid State Commun., 2012, 152, 1341.

7 F. Xia, T. Mueller, Y. Lin, A. Valdes-Garcia and P. Avouris, Nat. Nanotechnol., 2009, 4, 839.

8 C. Liu, Y. Chang, T. B. Norris and Z. Zhong, Nat. Nanotechnol., 2014, 9, 273.

9 X. Xu, N. M. Gabor, J. S. Alden, A. M. Zande and P. L. McEuen, Nano Lett., 2010, 10, 562.

10 F. Xia, U. Yan and P. Avouris, Proc. IEEE, 2013, 101, 1717.

11 A. K. Geim and K. S. Novoselov, Nat. Mater., 2007, 6, 183.

12 A. K. Geim, Science, 2009, 324, 1530.

13 S. Pang, Y. Hernandez, X. Feng and K. Mullen, Adv. Mater., 2011, 23, 2779.

14 Y. Xu and J. Liu, Small, 2016, 12, 1400.

15 J. Meng, H. Song, C. Li, Y. Jin, L. Tang, D. Liu, Z. Liao, F. Xiu and D. Yu, Nanoscale, 2015, 7, 11611.

16 T. H. Bointon, G. F. Jones, A. D. Sanctis, R. H. Pearce, M. F. Craciun and S. Russo, Sci. Rep., 2015, 5, 16464.

17 B. Guo, L. Fang, B. Zhang and J. R. Gong, Insci. J., 2011, 1, 80. 18 H. Liu, Y. Liu and D. Zhu, J. Mater. Chem., 2011, 21, 3335.

19 R. Beams, L. G. Cancado and L. Novotny, J. Phys.: Condens. Matter, 2015, 27, 083002.

20 C. Stampfer, F. Molitor, D. Graf, K. Ensslin, A. Jungen and C. H. Wirtz, Appl. Phys. Lett., 2007, 91, 241907.
21 C. Casiraghi, S. Pisana, K. S. Novoselov, A. K. Geim and A. C. Ferrari, Appl. Phys. Lett., 2007, 91, 233108.

22 P. A. Khomyakov, G. Giovannetti, P. C. Rusu, G. Brocks, J. Brink and P. J. Kelly, Phys. Rev. B, 2009, 79, 195425.

23 G. Giovannetti, P. A. Khomyakov, G. Brocks, V. M. Karpan, J. Brink and P. J. Kelly, Phys. Rev. Lett., 2008, 101, 026803.

24 W. X. Wang, S. H. Liang, T. Yu, D. H. Li, Y. B. Li and X. F. Han, J. Appl. Phys., 2011, 109, 07 C501.

25 A. Kasry, M. A. Kuroda, G. J. Martyna, G. S. Tulevski and A. A. Bol, ACS Nano, 2010, 4, 3839.

26 J. Oh, K. Kim and G. Yeom, J. Nanosci. Nanotechnol., 2014, 14, 1120.

27 J. Shim, C. H. Lui, T. Y. Ko, Y. Yu, P. Kim, T. R. Heinz and S. Ryu, Nano Lett., 2012, 12, 648.

28 H. E. Romero, P. Joshi, A. K. Gupta, H. R. Gutierrez, M. W. Cole, S. A. Tadigadapa and P. C. Eklund, Nanotechnology, 2009, 20, 245501.

29 F. Schedin, A. K. Geim, S. V. Morozov, E. W. Hill, P. Blake, M. I. Katsnelson and K. S. Novoselov, Nat. Mater., 2007, 6, 652.

30 E. H. Hwang, S. Adam and S. D. Sarma, Phys. Rev. B, 2007, 76, 195421.

31 T. O. Wehling, K. S. Novoselov, S. V. Morozov, E. E. Vdovin, M. I. Katsnelson, A. K. Geim and A. I. Lichtenstein, Nano Lett., 2008, 8, 173.

32 D. Wei, Y. Liu, Y. Wang, H. Zhang, L. Huang and G. Yu, Nano Lett., 2009, 9, 1752.

33 H. Liu, Y. Liu and D. Zhu, J. Mater. Chem., 2011, 21, 3335.

34 A. Reina, X. Jia, J. Ho, D. Nezich, H. Son, V. Bulovic, M. S. Dresselhaus and J. Kong, Nano Lett., 2009, 9, 30.

35 S. Costa, J. Weis, O. Frank, M. Fridrichova and M. Kalbac, RSC Adv., 2016, 6, 72859.

36 J. Lee, G. Ahn, J. Shim, Y. Lee and S. Ryu, Nat. Commun., 2012, 3, 1024.

37 Y. Kang, J. Kang and K. J. Chang, Phys. Rev. B: Condens. Matter Mater. Phys., 2008, 78, 115404.

38 L. Zheng, X. Cheng, D. Cao, Z. Wang, C. Xia, Y. Yu and D. Shen, Appl. Phys. Lett., 2014, 104, 023112.

39 Y. M. Lin, H. Y. Chiu, K. A. Jenkins, D. B. Farmer, P. Avouris and A. Valdes-Garcia, IEEE Electron Device Lett., 2010, 31, 68. 